\title{
Caso de estudio: la auditoría administrativa aplicada en una empresa
}

\section{Case study: the administrative audit applied in a company}

Galo Mauricio Durán Salazar

Flérida Alcívar Cedeño

Pedro Ufredo León Cedeño

Shirley Janeth Vélez Alcívar

Universidad de Guayaquil, Ecuador

Autor para correspondencia: galo.duransa@ug.edu.ec, flerida.alcivarced@ug.edu.ec, pedro.leonce@ug.edu.ec, shirleyvelezt@gmail.com

Fecha de recepción: 21 de Enero de 2018 - Fecha de aceptación: 25 de julio de 2018

Resumen: La investigación tiene como objetivo mostrar la metodología y resultados de una auditoria administrativa realizada en una empresa que se denominó "caso de estudio", en este caso se buscó determinar el nivel de cumplimiento que tiene la empresa con los organismos de control. La importancia de una auditoria de este tipo, implica revisar los procesos administrativos y contables a fin de detectar falencias que permitan hacer correcciones y favorezcan la continuidad del negocio. La investigación fue de tipo documental, utilizó como instrumentos entrevistas a personal clave de la empresa, revisión de documentos facilitados por la empresa, y la metodología COSO. Los resultados de la investigación indicaron que la empresa caso de estudio tuvo un nivel de confianza del $66 \%$ lo cual es moderado, generando un nivel de riesgo del 34\%. Las falencias detectadas fueron que no existen manuales de funciones específicas para los empleados y que se mesclan las actividades que realizan los trabajadores, lo cual es un riesgo, ante la falta de responsabilidades en el personal. Otra situación que se presentó fue que la gerencia y el área financiera tienen poca comunicación y el área responsable de la empresa termina siendo el financiero. Estas dos situaciones se recomendaron deben ser resueltas para el mejoramiento del negocio.

Palabras Claves: auditoria administrativa; COSO; cuestionarios; nivel de confianza

Abstract: The objective of the research is to show the methodology and results of an administrative audit done in a company that in this case was called "case study", it was tried to determine the level of compliance that the company has with the control organisms. The importance of an audit of this type involves reviewing the administrative and accounting processes in order to detect weaknesses that allow corrections and favor the continuity of the business. The investigation was of documentary type, used as instruments interviews to key personnel of the company, review of documents facilitated by the company, and the methodology COSO. The results of the research indicated that the company's case study had a confidence level of $66 \%$ which is moderate, generating a level of risk of $34 \%$. The shortcomings detected were that there are no specific function manuals for employees and that the activities carried out by the workers are combined, which is a risk, given the lack of responsibilities on the part of the staff. Another situation was that management and the financial area have little communication and the area responsible for the company ends up being the financial area. These two situations are recommended should be resolved for the improvement of the business.

Key Words: administrative audit; COSO; questionnaires; confidence level 


\section{Introducción}

La empresa analizada en la presente investigación se denomina "caso de estudio", es una compañía que se dedica a la importación y venta de productos médicos para traumatólogos y odontólogos creados en el año 2011, se encuentra ubicada en la ciudad de Guayaquil. La empresa ha ido creciendo en los pocos años que tiene en el mercado, como toda organización necesita de metas, para ello, es necesario una planificación financiera que permita la asignación de recursos basado en los objetivos del negocio, en otras palabras, se requiere de una organización integral; por ello, la gerencia solicitó una auditoria administrativa que evalué sí sus políticas y procedimientos son óptimos, si se cumple con los organismos de control de manera oportuna y evalué la forma en que la propia administración ha dirigido el negocio.

Según Godínez (2011) afirmó que la Auditoría Administrativa revisa los procesos administrativos, busca la detección de fallas dentro de una organización a fin de corregirlas y reducir los riesgos como tal; evalúa las decisiones tomadas por la gerencia dentro de una empresa, en este caso sobre su estructura, políticas, procesos, sistemas de información, entre otras áreas; considerando que los recursos son escasos y se deben tomar las decisiones óptimas en la organización.

Franklin (2007) indicó que es una revisión analítica o parcial de las organizaciones, con el objetivo de precisar el nivel de eficiencia que tienen la empresa y determinar las oportunidades que tienen para mejorar en sus procesos y procedimientos, a fin de incrementar valor a los servicios y actividades que brinda. Entre las ventajas de realizar una auditoria esta realizar intercambio de conocimientos con expertos, detectar inconsistencias, problemas generando soluciones de forma rápida, contribuir al logro de los objetivos de crecimiento del negocio.

Para las compañías, es importante contar con una adecuada administración de los recursos, ya que muchas de ellas fracasan antes de cumplir cinco años en el mercado, debido a un deficiente control interno que le dé la opción de validar el cumplimiento de los objetivos a corto, mediano y largo plazo.

Según Rodríguez (2011) en el país, las empresas deben cumplir con los requerimientos que exigen las autoridades de control, entre ellos el Servicio de Rentas Internas (SRI), Instituto Ecuatoriano de Seguridad Social (IESS) y distintos Ministerios, por ello la empresa caso de estudio, se planteó la situación que se observa en la figura 1 sobre el cumplimiento que ha realizado la organización durante el año 2016: 


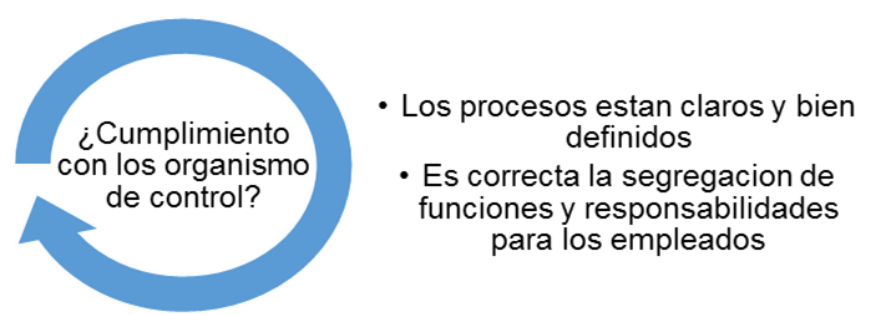

Figura 1 Cumplimiento en la empresa

Para Jiménez (2010) el riego de fraude es alto en las organizaciones, en la medida que no existan controles adecuados y no se los evalúen a tiempo, por ello, la aplicación de una auditoría administrativa, permite anticipar problemas en una empresa y tomar correctivos que permitan la continuidad del negocio; por ello, la empresa caso de estudio se planteó la siguiente pregunta ¿De qué manera la realización de una Auditoría Administrativa contribuye a la toma de decisiones?.

Por lo anterior, se procede a presentar la auditoria administrativa realizada a la empresa, a fin de contribuir con mejoras, en base a los hallazgos encontrados y mostrar un esquema que puede ser utilizado por profesionales en el área de la auditoria.

\section{Métodos}

La presente investigación aplicó el método del estudio de caso, y revisión documental de información de la empresa. Los instrumentos utilizados fueron la entrevista a personal clave del negocio en este caso gerencia y finanzas, y la observación-análisis de los documentos recibidos por parte de organización, se usó cuestionarios para la aplicación de la herramienta COSO I, necesarios para la auditoria, que se realizó en 60 horas, conforme la programación presentada en la tabla 1:

Tabla 1 Planificación de la auditoria

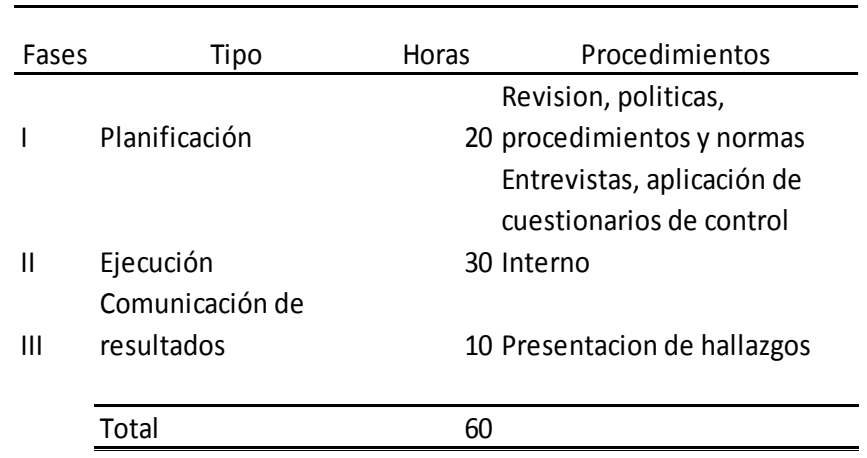

\section{Planificación}

La revisión de políticas, procedimientos y normas en la empresa caso de estudio, indicaron que tiene un buen posicionamiento en el mercado, sin embargo, las políticas contables tienen problemas en aplicarse correctamente como por ejemplo las compras que se realizan con el fondo de caja chica y reembolsos de gastos, no se entregan en las fechas respectivas y de esta 
manera no se utilizan para la declaración de impuestos de IVA y retenciones en la fuente, de esta forma el gasto se convierte en no deducible para el cálculo del impuesto a la renta; además los controles observados son débiles y deben ser corregidos.

\section{Ejecución}

En la etapa de ejecución de la auditoría se validó las funciones que realizan los empleados en relación a las actividades que le corresponde a cada trabajador. Luego se aplicó un cuestionario sobre controles internos para determinar si realmente se cumple o no con los controles establecidos. En base a los resultados de las entrevistas a personal clave de la empresa y análisis de documentos recibidos por parte de la empresa se determinó que:

- Los manuales de funciones del personal administrativos no fueron proporcionados, sin embargo, se mencionó que sí existen.

- Se recopiló políticas implementadas por la empresa, respecto al manejo del inventario y reposición del mismo, estableciendo que los controles utilizados son vulnerables, en vista, que no existe un inventario físico durante el año 2016.

- Los procesos de compras y ventas no se encuentran registrados en un manual, siendo necesario la creación de un manual de procedimientos internos.

- Sobre el cumplimiento con lo que indica la Ley de Discapacidades, la empresa no contrató empleados con discapacidad a pesar de tener más de 25 trabajadores: la ley obliga a realizarlo por cada 25 trabajadores tener 1 empleado con discapacidad.

- Los pagos al IESS, algunos meses se realizó con retrasos, además algunos trabajadores no fueron afiliados desde el primer día de labores. Los pagos al SRI, se realizaron a tiempo.

- No se aplica una política de cobranzas, no hay evidencia de esto, lo puede generar iliquidez y retrasos en los pagos que debe realizar la empresa con trabajadores, proveedores, importaciones y cumplimiento con los organismos de control.

\section{Aplicación de cuestionarios}

En la tabla 2 se presenta el cuestionario de preguntas utilizado, con la finalidad de establecer el tipo de controles que se ha manejado dentro de la compañía, esto determinó el nivel de riesgo y confianza que existen en los procesos administrativos, manejo de efectivo y de cumplimiento de políticas por parte del personal en la empresa. 
Tabla 2 Cuestionario de Controles

\begin{tabular}{|c|c|c|c|}
\hline Descripción & $\mathrm{Si}$ & No & Observación \\
\hline $\begin{array}{l}\text { El personal contratado posee funciones } \\
\text { separadas y segregadas de acuerdo a sus puestos } \\
\text { de trabajo? }\end{array}$ & & $\mathrm{X}$ & $\begin{array}{l}\text { A pesar de que la administración alega que existen } \\
\text { manuales de funciones, no se observa segregación de } \\
\text { funciones claras, considerando el tipo de estructura } \\
\text { que llevan. }\end{array}$ \\
\hline $\begin{array}{l}\text { ¿De qué manera se realizan los cobros a los } \\
\text { zlientes? }\end{array}$ & & $\mathrm{X}$ & $\begin{array}{l}\text { Se otorga crédito dependiendo del cliente: } 15,30,45 \text {, } \\
60 \text { y } 90 \text { días, sin embargo estos plazos no se cumplen } \\
\text { por la falta de seguimiento, lo que causa una cartera } \\
\text { por cobrar con un alto índice de morosidad. }\end{array}$ \\
\hline $\begin{array}{l}\text { ¿Si coinciden los valores que pagan los clientes vs } \\
\text { il saldo por cobrar en contabilidad? }\end{array}$ & $\mathrm{X}$ & & $\begin{array}{l}\text { Los registros contables síse encuentran al día, ya que } \\
\text { la información se ingresa de forma constante. }\end{array}$ \\
\hline $\begin{array}{l}\text { ¿Cómo se aprueban las compras y pagos a } \\
\text { proveedores? }\end{array}$ & $\mathrm{X}$ & & $\begin{array}{l}\text { Las compras de activos son aprobadas por la Gerente } \\
\text { General, quien es la firma autorizada en bancos. Y el } \\
\text { resto de las compras por el Gerente Financiero. Se } \\
\text { sacan pagos a proveedores dos veces al mes por } \\
\text { temas de flujo y de firma de la Gerente General. }\end{array}$ \\
\hline ¿La contabilidad se mantiene actualizada? & $\mathrm{X}$ & & $\begin{array}{l}\text { Toda la información se mantiene ingresada de forma } \\
\text { diaria. }\end{array}$ \\
\hline $\begin{array}{l}\text { ¿Cuáles son los gastos de caja chica? ¿Se } \\
\text { ingresan los documentos a tiempo? }\end{array}$ & & $\mathrm{X}$ & $\begin{array}{l}\text { El fondo rotativo de caja chica se estableció con el fin } \\
\text { de cubrir gastos menores, el problema radica al } \\
\text { momento del ingreso de las facturas debido a que por } \\
\text { los retrasos en las entregas de información se ingresan } \\
\text { de manera tardía. }\end{array}$ \\
\hline $\begin{array}{l}\text { ¿La compañía tiene procedimientos para detectar } \\
\text { faltantes de caja o fraudes laborales? }\end{array}$ & $\mathrm{X}$ & & $\begin{array}{l}\text { Los faltantes de caja pueden ser fácilmente } \\
\text { detectables gracias al control del flujo que se maneja. }\end{array}$ \\
\hline ¿Quiénes tienen trato directo con los clientes? & $\mathrm{X}$ & & Los clientes tratan directamente con los vendedores. \\
\hline $\begin{array}{l}\text { ¿De qué manera se realizan los anticipos de } \\
\text { sueldos y cómo se descuentan? }\end{array}$ & $\mathrm{X}$ & & $\begin{array}{l}\text { Los anticipos de sueldos se otorgan de acuerdo al } \\
\text { flujo con el que se encuentre la compañía y son } \\
\text { aprobados por el Gerente Financiero. }\end{array}$ \\
\hline $\begin{array}{l}\text { ¿Se evalúa al personal operativo en cuanto a su } \\
\text { rendimiento? }\end{array}$ & $\mathrm{X}$ & & No se evalúa al personal operativo. \\
\hline
\end{tabular}

Posterior a ello, se presenta en la tabla 3 , los documentos de divulgaciones que permitieron corroborar los controles existentes del negocio, sobre los cuales se emitirán comentarios.

\begin{tabular}{lcc} 
Tabla 3 Documentos para divulgación de información \\
\hline \multicolumn{1}{c}{ Descripción } & Si hay & No hay \\
\hline \hline Funciones separadas, segregadas & & $\mathrm{X}$ \\
Registros contables al día & $\mathrm{X}$ & $\mathrm{X}$ \\
Control de caja chica & & $\mathrm{X}$ \\
Cobros a clientes & & \\
Cobros vs contabilidad & $\mathrm{X}$ & \\
Trato con clientes & $\mathrm{X}$ & \\
Aprobaciones de compras y pagos & $\mathrm{X}$ & \\
Políticas anti-fraudes & $\mathrm{X}$ & \\
Políticas de anticipos & $\mathrm{X}$ & \\
Evaluación de desempeño & $\mathrm{X}$ & \\
\hline
\end{tabular}

\section{Aplicación de la herramienta COSO}


El uso de la herramienta COSO, requiere detectar los riesgos significativos que se pueden presentar en cuanto al procedimiento administrativo, para así ejecutarlo en la compañía, conforme los cuestionarios de control que se aplicaron a los cinco componentes de control interno. De esta forma en la tabla 4 se presentan los riesgos significativos detectados.

Tabla 4 Riesgos Significativos

\begin{tabular}{clc}
\hline N. & Riesgos significativos & Aseveraciones \\
\hline 1 & Cobro de ventas & Integridad \\
2 & Asientos contables & Corte / Exactitud / Integridad \\
3 & Caja chica & Corte / Exactitud / Integridad \\
\hline
\end{tabular}

Para determinar los factores de riesgo que afectan a la empresa, y según lo que se pudo analizar en las entrevistas y la observación del ambiente de trabajo se estableció una puntuación con relación al nivel de confianza de 100 puntos para cada uno de los componentes del sistema COSO, la formula se presenta a continuación:

$$
\mathrm{NC}=\underline{\mathrm{CT}} \underset{\mathrm{PT}}{\mathrm{X}} 100
$$

Donde:

$\mathrm{NC}=$ Nivel de confianza

$\mathrm{CT}=$ Calificación total

PT= Puntuación total

Para determinar el nivel de confianza NC y el riego R de la empresa, en la tabla 5, se presenta los rangos que determinan $\mathrm{NC}$, que son alto bajo y moderado, conforme lo establece la técnica. Esta tabla permite determinar en qué rango esta la empresa luego de aplicar y tabular los cuestionarios respectivos.

Tabla 5 Riesgo y confianza

\begin{tabular}{ccc}
\hline Rango & riesgo & confianza \\
\hline $15 \%-40 \%$ & Bajo & Bajo \\
$41 \%-85 \%$ & Moderado & Moderado \\
$86 \%-95 \%$ & Alto & Alto \\
\hline
\end{tabular}

La evaluación del ambiente de control se dio en base a la estructura organizacional, manual de funciones, misión, visión, objetivos corporativos. Se evaluó sobre 30 puntos y se obtuvo una calificación de 22/30; siendo entonces el nivel de confianza NC del 73\%, este resultado es alto.

La evaluación del ambiente de control se dio en base a la estructura organizacional, manual de funciones, misión, visión, objetivos corporativos. Se evaluó sobre 30 puntos y se obtuvo una calificación de 22/30; siendo entonces el nivel de confianza NC del 73\%, este resultado es alto. 
La evaluación de las actividades de control, se realizó en base a parámetros como supervisión de la gerencia, control de actividades del personal, nivel de autorizaciones. Se evaluó sobre 25 , siendo el resultado 17/25, esto significó un NC del $68 \%$, que se considera moderado.

Para la evaluación de los riesgos, se analizó las operaciones administrativas de la empresa en funciones no segregadas, capacitación del personal, manuales de procedimientos contables y políticas contables. Esta evaluación se puntuó sobre 30, siendo el resultado de 22/30, de esta forma el NC del $73 \%$.

La evaluación de la comunicación en la organización, analizo como fluye la información entre gerencia y el personal, la empresa con sus clientes y proveedores. Se puntuó sobre 20, siendo el resultado de 13/20, esto significó un NC del $65 \%$.

Para la evaluación de la supervisión y el monitoreo, se revisaron temas como líneas de supervisión, evaluación de desempeño a los empleados, entre otros. Se puntuó sobre 20 y el resultado fue 10/20, es decir, un 50\% de NC.

En base a los resultados indicados anteriormente se presenta la tabla 6 que permite observar el nivel de confianza de la empresa en base a los componentes de control interno, que en este caso fue del $66 \%$, lo que significó un $34 \%$ de riesgo.

Tabla 6 Evaluación del nivel de confianza

\begin{tabular}{lcccc}
\hline \multicolumn{1}{c}{ Componentes } & $\begin{array}{c}\text { Calificación } \\
\text { confianza }\end{array}$ & Alta & Moderada & Baja \\
\hline Ambiente de control & $73 \%$ & $\mathrm{X}$ & & \\
Actividades de control & $68 \%$ & & $\mathrm{X}$ & \\
Evaluación de riesgos & $73 \%$ & $\mathrm{X}$ & & \\
Información y comunicación & $65 \%$ & & $\mathrm{X}$ & \\
Supervisión y monitoreo & $50 \%$ & & $\mathrm{X}$ & \\
\multicolumn{1}{c}{ TOTAL } & $\mathbf{6 6 \%}$ & & \\
\hline
\end{tabular}

\section{Cumplimiento de las politicas internas}

Según lo revisado durante la ejecución de la auditoría la empresa caso de estudio ha cumplido en lo relacionado a los siguientes aspectos:

- Cumplimiento con el Servicio de Rentas Internas

- Cumplimiento con el Seguro Social

- Cumplimiento con el Ministerio de Relaciones Laborales.

- Cumplimiento para desaduanizar la mercadería importada.

Entre los aspectos que no ha podido cumplir son los siguientes:

- Ley de discapacidades

- Anexo de ventas a crédito con Superintendencia de Compañías, que está relacionado con la cobranza a los clientes. 
De las seis políticas administrativas, que tienen que ver con el cumplimiento con los organismos de control, el porcentaje corresponde al $67 \%$.

\section{Análisis de los procedimientos aplicados y sus resultados}

Los procedimientos utilizados para la validación de la información han sido los siguientes: entrevistas, observaciones, revisión de documentación física,

En las entrevistas realizadas a la Gerente General y Gerente Financiero, se han podido determinar que existe una clara falta de supervisión y comunicación oportuna de las situaciones que ocurren en la compañía, es por ello que se denota la falta de una línea de supervisión directa que permita al personal sentirse seguro de a quién debe reportar sus actividades.

En cuanto a la revisión de la documentación importante de la empresa, se han leído las actas de la Junta General de Accionistas, escrituras de constitución, y otros documentos relevantes que mencionan los fines para los cuales ha sido creada la compañía.

Por medio de la observación de estos documentos y de los procesos que se ejecutan a través de las actividades ordinarias en la empresa, se ha podido evidenciar que no existen funciones segregadas, por lo tanto, existe el riesgo de algún fraude laboral por la falta de supervisión.

\section{Etapa de comunicación de resultados}

Los resultados obtenidos se presentan en la tabla 7 y 8 considerados como hallazgos 1 y 2 correspondientes a las actividades de control y supervisión, con la respectiva recomendación.

Tabla 7 Hallazgo 1

ACTIVIDADES DECONTROL

\begin{tabular}{|c|}
\hline ACTIVIDADES DECONTROL \\
\hline CRITERIO \\
\hline $\begin{array}{l}\text { El NC del } 73 \% \text {, indica un riesgo del } 27 \% \text {, lo } \begin{array}{l}\text { La falta de funciones especificas para los } \\
\text { empleados, provoca una carga laboral ineficiente, } \\
\text { cual significa una baja probabilidad de } \\
\text { riesgo para el negocio } \\
\text { lo que puede generar conflictos entre trabajadores }\end{array} \\
\text { y aumentos de riesgos en los procesos }\end{array}$ \\
\hline Ausencia de manual de funciones. \\
\hline Funciones mezcladas \\
\hline RECOMENDACIÓN \\
\hline $\begin{array}{l}\text { Crear el manual de funciones, perfiles, cargos y difundirlos entre en el personal administrativo, } \\
\text { comercial y de operaciones. }\end{array}$ \\
\hline
\end{tabular}

Tabla 8 Hallazgo 2 


\begin{tabular}{l}
\hline \multicolumn{1}{c}{ SUPERVISIÓN Y MONITOREO } \\
$\begin{array}{l}\text { Las lineas de supervision son debiles, por La gerencia financiera y general estan al mismo nivel, no } \\
\text { ello existe un riesgo del 50\% } \\
\text { CAtan claras las autorizaciones }\end{array}$ \\
\hline$\frac{\text { CRITERIO }}{\text { Eusencia de supervisión y autorizaciones. }}$ \\
$\frac{\text { Riesgo sobre la administración. }}{\text { RECOMENDACIÓN }}$ \\
\hline $\begin{array}{l}\text { Se debe implementar politica de autorizaciones y supervisión, así como la creación de un departamento } \\
\text { de auditoría interna. }\end{array}$ \\
\hline
\end{tabular}

\section{Conclusiones}

La empresa caso de estudio, es nueva en el mercado, a pesar de ello ha ido creciendo de manera importante en el sector de insumos médicos, por ello, la gerencia decidió que se realizara una auditoria administrativa que evalué los procesos administrativos y contables, a fin de tomar medidas que permitan hacer correcciones.

La auditoría realizada reveló que la política de cumplimiento con los organismos de control corresponde al $67 \%$, en vista que no cumplió con 2 entidades, una el ministerio de relaciones laborales en cuanto a la contratación de personal con discapacidad y segundo la no presentación del anexo de ventas a crédito a la Superintendencia de Compañías.

La aplicación del COSO, mostró un 66\% de nivel de confianza, el cual es moderado y el nivel del riesgo corresponde al 34\%, en base a la información obtenida en los cuestionarios, por ello, las recomendaciones que se sugieren son crear manual de funciones específicas para los empleados a fin de evitar conflictos entre los trabajadores y potenciales riesgos de fraude, al no estar definidas las responsabilidades.

\section{Bibliografía}

Alexander y Jorge (2010). "Propuesta de un sistema de evaluación para fortalecer el desempeño laboral del personal en la empresa de distribuciones Olano S.A.C de la ciudad de Chiclayo 2010”. (Tesis). Chiclayo: Universidad Señor de Sipán.

Auditores, Contadores, y Consultores Financieros . (Agosto de 2015). auditoresycontadores.com. Obtenido de auditoresycontadores.com: http://www.auditoresycontadores.com/contabilidad/61-que-es-el-control-interno-ycuales-son-los-elementos.

Ávila, R. (1992). Introducción a la Metodología de la Investigación. Editorial Estudios y Ediciones RA. Lima - Perú.

Barry, T. (2001). "La excelencia como hábito, la calidad no es un objetivo, sino una estrategia". Editorial Panorama S.A. México.

Blinder, F. (2013). Conocer y evitar el fraude interno en la empresa. Obtenido de http://maestrofinanciero.com/conocer-y-evitar-el-fraude-interno-en-la-empresa/ 
Bradford, C. (1964). Manual de Auditoría Operacional.

Buchele (2009). Control Administrativo Disponible en:https://webcache.googleusercontent.com/search?q=cache:M7tT9IdGriUJ:https: //episunprg.files.wordpress.com/2009/09/gruponc2ba13el_control_administrativo.docx $+\& \mathrm{~cd}=4 \& \mathrm{hl}=\mathrm{es} \& \mathrm{ct}=\mathrm{clnk} \& \mathrm{gl}=\mathrm{pe}$

Chiavenato, I. (2006). Introducción a la Teoría General de la Administración.

Consejo Nacional de Discapacidades. (febrero de 2014). consejodediscapacidades.gob.ec. Obtenido de consejodediscapacidades.gob.ec: http://www.consejodiscapacidades.gob.ec/wpcontent/uploads/downloads/2014/02/ley_organica_discapacidades.pdf

Franklin, E. (2007). Auditoria administrativa: gestión estratégica del cambio. México: McGraw Hill Editorial.

Furlán (2008). Caracterización del control interno en el área de almacén en las empresas comerciales del Perú. Disponible en: http://www.monografias.com/trabajospdf5/caracterizacion-del-control-interno-areaalmacen-empresas-comerciales-delperu/caracterizacion-del-control-interno-areaalmacen empresas-comerciales-delperu2.shtml\#ixzz4MKFndi00

Gordillo. (2015). Concepto y naturaleza del procedimiento administrativo. Obtenido de http://www.gordillo.com/pdf_tomo5/03/03-capitulo1.pdf

Hernández , R., Fernández, C., \& Baptista, P. (2010). Metodología de la Investigación. México D. F, México: Mcgraw-Hill / Interamericana Editores, S.A. De C.V. .

Mendivil, M. (2005). Auditoría administrativa aplicada a una empresa de giro ferretero. Obtenido de http://biblioteca.itson.mx/dac_new/tesis/127_maria_mendivil.pdf

Tamayo, M. (2006). El proceso de la investigación científica. México: Editorial LIMUSA. 